\title{
Effect of Bursty Impulsive Noise on the Performance of Multi-relay DF Cooperative Relaying Scheme
}

\author{
Md. Sahabul Alam \\ Department of Electrical and Computer Engineering \\ McGill University, Montreal, Canada \\ Email: md.s.alam@mail.mcgill.ca
}

\author{
Fabrice Labeau \\ Department of Electrical and Computer Engineering \\ McGill University, Montreal, Canada \\ Email: fabrice.labeau@mcgill.ca
}

\begin{abstract}
In this paper, we consider the performance analysis of a decode-and-forward (DF) cooperative relaying (CR) scheme over bursty impulsive noise channel. As compared to existing literature, here, we generalize the performance analysis to the multi-relay scenario with and without considering error propagation from the relays. For this scheme, we evaluate the bit error rate (BER) performance in the presence of Rayleigh fading with a maximum a posteriori (MAP) receiver. From the obtained results it is seen that, similar to single relay scheme, the proposed MAP receiver attains the lower bound derived for multi-relay DF CR scheme also, and performs significantly better than the conventional schemes developed for additive white Gaussian noise (AWGN) channel and memoryless impulsive noise channel. Moreover, the performance improvement of optimal MAP receiver over the memoryless receivers is substantial with increasing the number of relays.
\end{abstract}

\section{INTRODUCTION}

Cooperative relaying (CR) is a method to exploit spatial diversity in wireless networks without necessitating the placement of multiple antennas at each node [1]. It is very attractive in some scenarios, for example, wireless sensor networks where the sensor nodes cannot afford multiple antennas due to their size and cost constraints. Although there exists a rich body of literature on CR schemes in various aspects, many of them are restricted to the AWGN assumption. However, practical wireless systems are impaired by non-Gaussian noise and interference also [2]. For example, due to partial discharge and switching effects impulsive noise is observed in some mobile radio scenarios [2], [3]. The pairwise error probability (PEP) of multi-relay amplify-and-forward (AF) CR scheme over flat fading channel in the presence of impulsive noise modeled by Middleton class-A [2] has been investigated in [4]. Simulation results demonstrated that the performance of CR schemes highly depends on the impulsive nature of the noise. Similar performance analysis is carried out in [5] for DF CR schemes. It is shown that similar to the Gaussian noise case, the system achieves full diversity order asymptotically with signal-to-noise ratio (SNR) in impulsive noise scenario. In [6], closed-form asymptotic error-rate expressions were derived for an AF CR scheme with multiple relays which is valid for arbitrary non-Gaussian noise and interference with finite moments. The simulation results reveal that, at high SNR, full diversity order is obtained and is independent of the type of noise. The authors in [7] studied the performance of direct transmission (DT) and single-relay DF CR scheme over flat Rayleigh fading and Bernoulli-Gaussian [8] impulsive noise. The obtained results showed that the optimal Bayes receiver [9] obtains an additional 3dB SNR gain over the MRC combiner by considering impulsive noise in the detection process.

However, all of the above performance analyses for CR schemes have been carried out over i.i.d. impulsive channels which cannot provide any information on noise time correlation, as observed in experimental measurements [3]. In this context, in [10] we provided a mathematical framework for the performance analysis of a single-relay DF CR scheme over bursty impulsive noise channel modeled by a two-state Markov-Gaussian (TSMG) [11] process. As an extension, in this paper, we generalize the performance analysis to the multirelay scenario. Two different relaying strategies are considered depending on the processing performed by the relay: simple DF relaying (SR) and selective DF relaying (SDFR). From the obtained results it is seen that as in the single relay scheme, the proposed optimal MAP receiver achieves the lower bound (LB) derived for multi-relay DF CR scheme and performs significantly better than the conventional schemes developed for AWGN channel and memoryless impulsive noise channel. Moreover, the performance improvement of optimal MAP receiver over the memoryless receivers increases with increasing the number of relays.

The rest of the paper is organized as follows. In Section II, the system model is introduced. In Section III, we provide the mathematical framework of MAP decoding for the proposed scenario. Section IV provides the performances in terms of BER and finally, some conclusions are drawn in Section V.

\section{SYSTEM MODEL}

Here, we consider a DF CR scheme with $M$ relays, as shown in Fig 1. We assume that all nodes are equipped with a single antenna and share the same bandwidth for data transmission. We also assume that each node operates in halfduplex mode. The cooperative communication takes place in $M+1$ time slots, with normalized time intervals from $t_{0}$ to $t_{M}$. In the first time slot, $s$ transmits the data to $d$, and due to the broadcast nature of the wireless channel, the relays also receive it. Each relay then demodulates and decodes the received signal to recover the source information and either retransmits, or declares that it will remain silent. During this period, $s$ remains in the silent mode as indicated by the dotted line in Fig 1. For simple DF relaying, the relays always retransmit the decoded data to the destination. Hence, the decoded data with possible errors are forwarded from the relays to the destination. It is different from most papers on multi-relay DF CR schemes where it is typically assumed that if any of the participating 

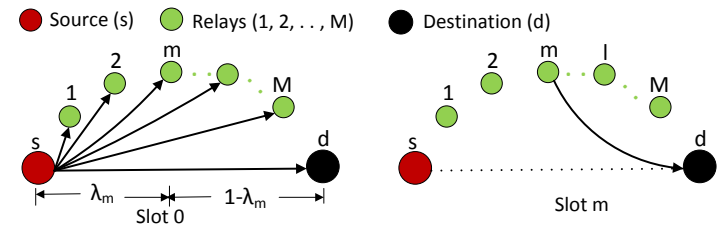

Fig. 1. Cooperative communication with half-duplex relaying.

relays decodes the source message perfectly it will forward its decoded information to the destination, otherwise it will stay in silent mode, i.e., what we call selective DF relaying, which is decided by comparing the received SNR at the relay to a given threshold. However, in practical relaying systems an arbitrary chosen threshold does not guarantee error-free detection and hence decoding errors may occur at the relay [12]. Although this problem could be solved by considering CRC checking at the relays, it is bandwidth consuming and induces extensive overhead since CRC checking usually takes place at the MAC layer. To avoid this, our analysis remains more general and considers that decoding error might be propagated by the relays.

Consider that the source $s$ generates a frame of binary information of length $K$ bits $\left(b_{0}, b_{1}, \ldots, b_{K-1}\right)$, mapped into a BPSK modulated sequence $\left(x_{s, 0}, x_{s, 1}, \ldots, x_{s, K-1}\right)$, and transmitted to both $m, m \in\{1,2, \ldots, M\}$, and $d$ in the first time slot. The signals received at $m$ and $d$ at each time epoch $k, k=0,1, \ldots, K-1$ can be respectively expressed as

$$
\begin{aligned}
y_{s m, k} & =\sqrt{\mathrm{P}_{s}} h_{s m, k} x_{s, k}+n_{s m, k}, \\
y_{s d, k} & =\sqrt{\mathrm{P}_{s}} h_{s d, k} x_{s, k}+n_{s d, k},
\end{aligned}
$$

where $\mathrm{P}_{s}$ is the average source transmission power, $h_{i j, k}$ is the channel coefficient for the $i j$ link, $i \in(s, m)$ and $j \in(m, d)$, and $n_{i j, k}$ is the noise term for the $i j$ link that captures the combined effects of AWGN and the impulsive interferers. We assume independent Rayleigh fading in all links, i.e., for each ij link, $h_{i j}$ is modeled as a zero-mean, independent, circularly symmetric complex Gaussian random variable with variance $\Omega_{i j}^{2} \equiv \varepsilon\left\{\left|h_{i j}\right|^{2}\right\}=1 / \lambda_{i j}^{\eta}$, where $\varepsilon\{\cdot\}$ denotes expectation operator, $\lambda_{i j}$ is the relative distance of $i$ from $j$, and $\eta$ is the path loss exponent. It is assumed that the channel coefficients are known by the receiver side, but not by the transmitter side. The noise sample $n_{i j, k}$ is modeled as a TSMG process, which is in fact a Markov process in with the marginal distribution in each state are Gaussian. We assume that for each $i j$ link, $n_{i j, k}$ follows a zero-mean, independent, circularly symmetric complex Gaussian distribution with variances depending on the noise state $s_{k}, s_{k} \in\{G, B\}$. In our noise modeling, $G$ stands for the good channel that is impaired by the background Gaussian noise only and $B$ for the bad channel which is impaired by impulsive interferers also.

In slot 1 , the received signal $y_{s 1}$ at the first relay is passed through a demodulator to recover the source information. After recovering the source information, the relay modulates it using the same modulation format as in $s$ and forwards it to the destination with average transmission power $\mathrm{P}_{1}$. Generally in slot $m$, the $m$-th relay transmits its decoded information to the destination with average transmission power $\mathrm{P}_{m}$. The signal received at the destination in this case is given by

$$
y_{m d, k}=\sqrt{\mathrm{P}_{m}} h_{m d, k} x_{m, k}+n_{m d, k}
$$

where $x_{m, k}$ is the transmitted signal from $m$ which can be defined as $x_{m, k}=x_{s, k} \oplus e_{m, k}$, where $\oplus$ indicates modulo 2 addition and $e_{m, k}$ is a binary random variable representing the error event at the output of the relay decoder with a corresponding bit error probability $p\left(e_{m, k}=1\right)=\theta_{m}$. For fair comparison between DT and CR schemes, in our discussion we assume that the total source transmission power for direct transmission $\mathrm{P}_{T}$ is equal to $\mathrm{P}_{s}+\sum_{m=1}^{M} \mathrm{P}_{m}$.

\section{PERFORMANCE ANALYSIS}

\section{A. Maximum a Posteriori (MAP) Detection}

The MAP decoding rule at the destination is given by

$$
\hat{x}_{s, k}= \begin{cases}1 & \text { if } L_{k} \geq 0 \\ -1 & \text { if } L_{k}<0\end{cases}
$$

where, $\hat{x}_{s, k}$ is the estimate of the source's transmitted sequence $x_{s, k}$ and $L_{k}$ is the log-likelihood ratio (LLR). For a multi-relay DF CR scheme, since the transmission from the source to the relay links in the first slot is similar to the direct transmission from the source to the destination, $L_{k}$ at the $m$-th relay for the $s m$ link is obtained by [10]

$$
\begin{aligned}
& L_{s m, k}=\ln \left\{\frac{p\left(x_{s, k}=1, y_{s m}^{K}\right)}{p\left(x_{s, k}=-1, y_{s m}^{K}\right)}\right\}=\ln \left\{\frac{p\left(x_{s, k}=1\right)}{p\left(x_{s, k}=-1\right)} \times\right. \\
& \left.\frac{\sum_{s_{k}, s_{k+1}} \alpha_{m, k}\left(s_{k}\right) \delta_{m, k}\left(x_{s, k}=1, s_{k}, s_{k+1}\right) \beta_{m, k+1}\left(s_{k+1}\right)}{\sum_{s_{k}, s_{k+1}} \alpha_{m, k}\left(s_{k}\right) \delta_{m, k}\left(x_{s, k}=-1, s_{k}, s_{k+1}\right) \beta_{m, k+1}\left(s_{k+1}\right)}\right\} .
\end{aligned}
$$

where $\alpha_{m, k}\left(s_{k}\right)$ and $\beta_{m, k}\left(s_{k}\right)$ are referred to as the forward and backward filters and $\gamma_{m, k}\left(x_{s, k}, s_{k}, s_{k+1}\right)$ represents the branch metrics of the trellis diagram, which are defined by

$$
\begin{aligned}
\alpha_{m, k}\left(s_{k}\right) & =p\left(y_{s m, 0}, y_{s m, 1}, \ldots, y_{s m, k-1}, s_{k}\right), \\
\beta_{m, k}\left(s_{k}\right) & =p\left(y_{s m, k}, y_{s m, k+1}, \ldots, y_{s m, K-1} \mid s_{k}\right), \\
\delta_{m, k}\left(x_{s, k}, s_{k}, s_{k+1}\right) & =p\left(s_{k+1} \mid s_{k}\right) p\left(y_{s m, k}-\sqrt{\mathrm{P}_{s}} h_{s m, k} x_{s, k} \mid s_{k}\right)
\end{aligned}
$$

The forward and backward filters can be computed recursively as

$$
\begin{aligned}
& \alpha_{m, k+1}\left(s_{k+1}\right)=\sum_{s_{k}, x_{s, k}} \alpha_{m, k}\left(s_{k}\right) p\left(x_{s, k}\right) \delta_{m, k}\left(x_{s, k}, s_{k}, s_{k+1}\right), \\
& \beta_{m, k}\left(s_{k}\right)=\sum_{s_{k+1}, x_{s, k}} \beta_{m, k+1}\left(s_{k+1}\right) p\left(x_{s, k}\right) \delta_{m, k}\left(x_{s, k}, s_{k}, s_{k+1}\right),
\end{aligned}
$$

where the forward and backward filters are initialized with $\alpha_{m, 0}\left(s_{0}=S\right)=p_{S}$ and $\beta_{m, K}\left(s_{K}=S\right)=1, S \in(G, B)$. In order to derive the analytical BER formula for the source-relay links, we assume that the receiver has the knowledge of the state $s_{k}$ and the variance of each state. Then, the average BER at the $m$-th relay is given by [10]

$P_{b, m}=p_{G}^{s m}\left(1-\sqrt{\frac{\bar{\gamma}_{s m}^{G}}{1+\bar{\gamma}_{s m}^{G}}}\right)+p_{B}^{s m}\left(1-\sqrt{\frac{\bar{\gamma}_{s m}^{B}}{1+\bar{\gamma}_{s m}^{B}}}\right)$.

where, $p_{G}^{s m}$ and $p_{B}^{s m}$ are the steady state probabilities of having in good state and bad state respectively, $\bar{\gamma}_{s m}^{w}=\frac{\mathrm{P}_{\mathrm{T}} \Omega_{s m}^{2}}{\sigma_{s m, w}^{2}}$ is the average received SNR of $s m$ link, $w \in(0,1) \equiv(G, B)$ and $\sigma_{s m, w}^{2}=R_{s m}^{w} \sigma_{G}^{2}$ is the noise variance of $n_{s m}$ with $R_{s m}$ is the impulsive to Gaussian noise power ratio for the $s m$ link. 


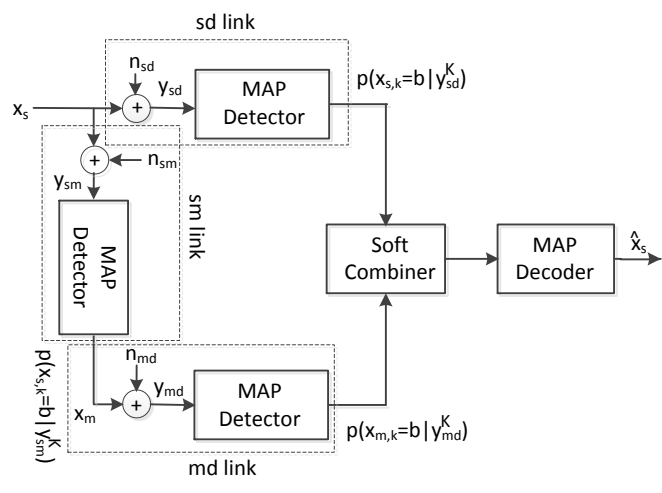

Fig. 2. MAP receiver for DF cooperative relaying over correlated impulsive noise. The system is composed of $2 M+1$ MAP detectors one for each link.

\section{B. BER of multi-relay DF cooperative relaying}

The end-to-end BER performance of multi-relay DF CR scheme depends on different relaying strategies such as simple $D F$ relaying in which the relays always re-transmit their decoded information to the destination in their time slots. The optimal MAP decoding under this condition becomes

$$
L_{d, k}=\ln \left\{\frac{p\left(x_{s, k}=1 \mid y_{s d}^{K}, y_{1 d}^{K}, y_{2 d}^{K}, \ldots, y_{M d}^{K}\right)}{p\left(x_{s, k}=-1 \mid y_{s d}^{K}, y_{1 d}^{K}, y_{2 d}^{K}, \ldots, y_{M d}^{K}\right)}\right\}
$$

Following the same procedure in [10], we have

$$
\begin{aligned}
L_{d, k}=\ln & \left\{\frac{p\left(x_{s, k}=1, y_{s d}^{K}\right)}{p\left(x_{s, k}=-1, y_{s d}^{K}\right)}\right\}+\sum_{m=1}^{M} \ln \left\{\frac{p\left(x_{m, k}=1, y_{m d}^{K}\right)}{p\left(x_{m, k}=-1, y_{m d}^{K}\right)}\right\} \\
& +\sum_{m=1}^{M} \ln \left\{\frac{1+\frac{\theta_{m}}{1-\theta_{m}}\left(\frac{p\left(x_{m, k}=-1, y_{m d}^{K}\right)}{p\left(x_{m, k}=1, y_{m d}^{K}\right)}\right)}{1+\frac{\theta_{m}}{1-\theta_{m}}\left(\frac{p\left(x_{m, k}=1, y_{m d}^{K}\right)}{p\left(x_{m, k}=-1, y_{m d}^{K}\right)}\right)}\right\}
\end{aligned}
$$

The first and second terms in (7) can be computed as (5) with the computation of $\alpha, \beta$, and $\delta$ for the $s d$ and $m d$ links. The third term can be estimated by knowing $\theta_{m}$ and the output of the MAP detectors for the $m d$ links. The whole operation is shown in Fig. 2.

On the other hand, for selective $D F$ relaying the average BER at the destination can be computed as [13]

$$
P_{b, S D F R}=\sum_{i=0}^{2^{M}-1} p(b, S D F R \mid \mathbf{V}=\mathbf{v}) \cdot p(\mathbf{V}=\mathbf{v})
$$

where, $p(b, S D F R \mid \mathbf{V}=\mathbf{v})$ is the error probability at the destination conditioned on a particular decoding state process and $p(\mathbf{V}=\mathbf{v})$ is the probability of happening that state process. In order to compute $p(b, S D F R \mid \mathbf{V}=\mathbf{v})$ we have to know which combiner is used for combining the signals coming from the source and the relays. For AWGN channel, i.e., when impulsive noise is absent, the maximum ratio combining (MRC) is optimal in the sense of minimizing the BER. The MRC combining is

$$
y_{d}=h_{s d}^{*} \sqrt{\mathrm{P}_{\mathrm{s}}} y_{s d}+\sum_{m=1}^{M} h_{m d}^{*} \sqrt{\mathrm{P}_{\mathrm{m}}} y_{m d} .
$$

When impulsive noise is present, the optimal MAP decoding of (7) for this scheme becomes

$L_{d, k}=\ln \left\{\frac{p\left(x_{s, k}=1, y_{s d}^{K}\right)}{p\left(x_{s, k}=-1, y_{s d}^{K}\right)}\right\}+\sum_{l=1}^{L} \ln \left\{\frac{p\left(x_{s, k}=1, y_{l d}^{K}\right)}{p\left(x_{s, k}=-1, y_{l d}^{K}\right)}\right\}$.

where, $L$ are the number of successful relays out of $M$ relays as indicated by the number of 1 's in the vector $\mathbf{v}$. In order to derive $P_{b, S D F R}(\mathbf{v})=p(b, S D F R \mid \mathbf{V}=\mathbf{v})$ it is assumed that the destination receiver has the knowledge of the states of $n_{s d}$ and $n_{l d}$, and the variances of each state. This makes the problem tractable and constitutes a LB on the actual BER. Under this consideration the optimal combiner is based on MRC. Let $\gamma_{0}^{u_{0}}=\frac{\mathrm{P}_{s}\left|h_{s d}\right|^{2}}{\sigma_{s d, u_{0}}^{2}}$ and $\gamma_{l}^{u_{l}}=\frac{\mathrm{P}_{l}\left|h_{l d}\right|^{2}}{\sigma_{l d, u_{l}}^{2}}$ are the instantaneous link SNRs of $s d$ and $l d$ links (for notation convenience, $l=0$ is used for the sd link), respectively, where $\sigma_{s d, u_{0}}^{2}=R_{s d}^{u_{0}} \sigma_{G}^{2}$ and $\sigma_{l d, u_{l}}^{2}=R_{l d}^{u_{l}} \sigma_{G}^{2}$ are the noise variances of $n_{s d}$ and $n_{l d}$, respectively, and $u_{0}, \ldots, u_{l} \in\{0,1\} \equiv(G, B)$. Since, $h_{s d} \sim C N\left(0, \Omega_{s d}^{2}\right)$ and $h_{l d} \sim C N\left(0, \Omega_{l d}^{2}\right)$, i.e., each link experience Rayleigh fading, $\gamma_{0}^{u_{0}}$ and $\gamma_{l}^{u_{l}}$ are exponentially distributed with mean $\bar{\gamma}_{0}^{u_{0}}$ and $\bar{\gamma}_{l}^{u_{l}}$. Then, conditioned on $\sigma^{2}=\left[\sigma_{s d, u_{0}}^{2} \sigma_{1 d, u_{1}}^{2} \ldots \sigma_{L d, u_{L}}^{2}\right], P_{b, S D F R}(\mathbf{v})$ is the BER of a $(L+1)$-branch MRC receiver in Rayleigh fading channel, which is given as [14]

$$
P_{b, S D F R}\left(\mathbf{v}, \sigma^{2}\right)=\frac{1}{2} \sum_{l=0}^{L} \pi_{l}\left[1-\sqrt{\frac{\bar{\gamma}_{l}^{u_{l}}}{1+\bar{\gamma}_{l}^{u_{l}}}}\right]
$$

where $\pi_{l}$ is defined as

$$
\pi_{l}=\prod_{\substack{j=0 \\ j \neq l}}^{L} \frac{\bar{\gamma}_{l}^{u_{l}}}{\bar{\gamma}_{l}^{u_{l}}-\bar{\gamma}_{j}^{u_{j}}}
$$

By averaging $P_{b, S D F R}\left(\mathbf{v}, \sigma^{2}\right)$ with respect to $\sigma^{2}$, we obtain the average BER for the cooperative link as

$$
\begin{gathered}
P_{b, S D F R}(\mathbf{v})=\frac{1}{2} \sum_{u_{0}=0}^{1} \ldots \sum_{u_{l}=0}^{1} \ldots \sum_{u_{L}=0}^{1}\left(p_{B}^{s d}\right)^{u_{0}}\left(p_{G}^{s d}\right)^{1-u_{0}} \\
\ldots\left(p_{B}^{l d}\right)^{u_{l}}\left(p_{G}^{l d}\right)^{1-u_{l}} \ldots\left(p_{B}^{L d}\right)^{u_{L}}\left(p_{G}^{L d}\right)^{1-u_{L}} P_{b, S D F R}\left(\mathbf{v}, \sigma^{2}\right) .
\end{gathered}
$$

Hence, the average BER at the destination is obtained by substituting (13) in (8).

\section{NUMERICAL RESUlTS}

Here, we present the BER performances of multi-relay DF $\mathrm{CR}$ schemes where a sequence of information bits of length 64,800 is transmitted over TSMG channels characterized by the identical parameters of bad state occurring rate $p_{B}=0.1$, channel memory $\gamma=100$, and impulsive to Gaussian noise power ratio $R=100$. In our simulations, it is assumed that the distance between the $s d$ pair is normalized to unity, i.e., $\lambda_{s d}=1$ and the relays are uniformly [13] distributed between the $s d$ pair with $d_{s 1}=d_{12}=\ldots=d_{M d}$. Also, slot durations $t_{0}=t_{1}=\ldots=t_{M}=1 / M+1$ and the path loss exponent $\eta=2$.

Fig. 3 shows the analytical and simulated BER performances of SDFR scheme with $M=2$ relays assuming different receiver structures. As a performance benchmark, the BER performance of DT is also shown. The proposed optimal MAP 


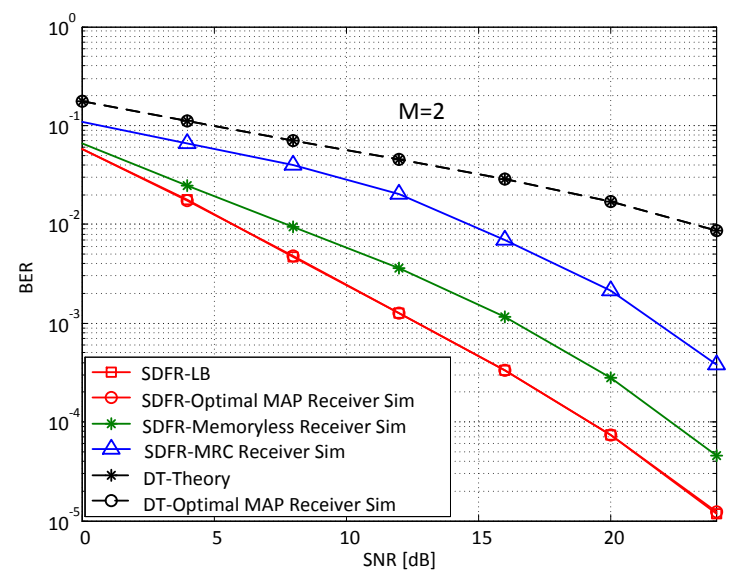

Fig. 3. Analytical and simulated BER performances of DT and SDFR scheme with $M=2$ relays. A system employing BPSK modulation is considered and the performance of various decoding schemes over TSMG channels, each characterized by $p_{B}=0.1, \gamma=100, R=100$ is shown.

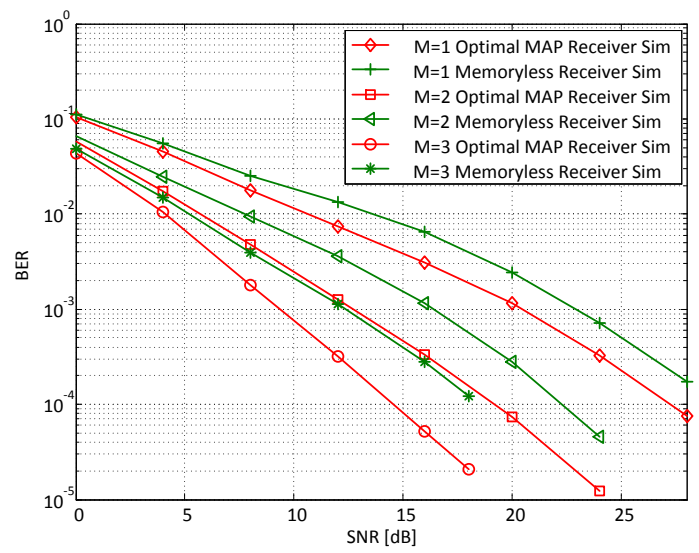

Fig. 4. BER performances of SDFR scheme against SNR with $M=1,2,3$ relays and different receiver structure at the destination. A system employing BPSK modulation is adopted and each channel is characterized by $p_{B}=0.1$, $\gamma=100$, and $R=100$ is shown.

receiver uses the MAP detection criterion, the memoryless receiver [9] is optimal for i.i.d. Bernoulli-Gaussian noise, and the MRC combiner [14] is optimal for AWGN channel. The BER formula in (8) is used to obtain the analytical results for SDFR scheme. To obtain the simulated BER, the LLR values for the source-relay links are obtained using (5) and the LLR values at the destination are obtained using (10). The BER performances are calculated for 2000 frames of 64,800 information bits each against $S N R$. The $S N R$ is defined as, $S N R=\varepsilon\left\{\left|x_{s, k}\right|^{2}\right\} / \sigma_{G}^{2}$, where $\sigma_{G}^{2}$ is the Gaussian noise power. From Fig. 3, it is seen that the analytical result perfectly matches with the simulation result. Also, our proposed MAP receiver achieves the LB derived for SDFR and performs significantly better than the MRC combiner and the memoryless receiver at the expense of higher complexity due to the MAP detection. This confirms the benefits of utilizing the noise memory in the detection process for multi-relay scenario.

Although similar conclusions hold for any number of cooperating relays, the performance gain provided by the utilization

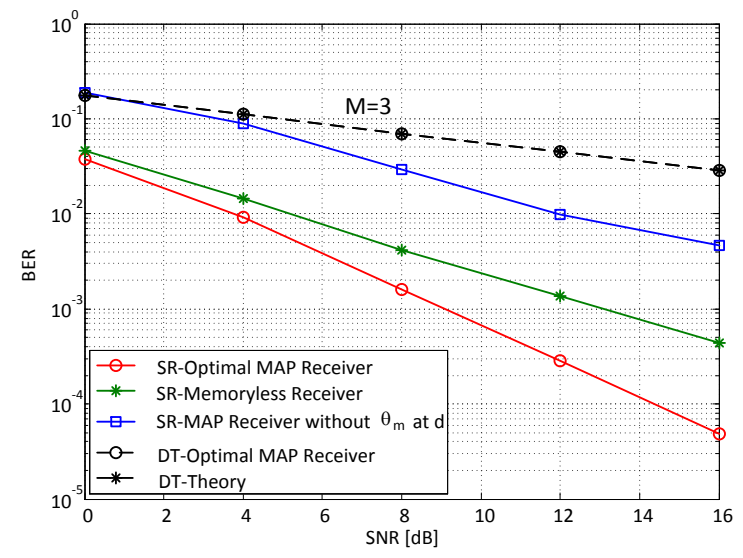

Fig. 5. BER performances of DT and SR scheme with $M=3$ relays and different realizations of $\theta_{m}$ at the destination. A BPSK modulation is considered and the performance of various decoding schemes over TSMG channels, each characterized by $p_{B}=0.1, \gamma=100, R=100$ is shown.

of memory depends on that value. This is shown in Fig. 4 for different realizations of $M$ while the other parameters remain unchanged. From the figure it is seen that as the value of $M$ increases, the performance of MAP receiver over the Memoryless receiver improves. This implies that the larger the number of cooperating relays are, the better the gain provided by the memory. The problem is the complexity of the MAP receiver which increases linearly with increasing the number of relays. Also, with $M$ relays the bandwidth efficiency is reduces to $1 / M+1$. From Fig. 4 it is also seen that keeping the total transmission power unchanged, as the number of cooperative relay increases, the BER performance improves for any type of receiving. While this is known for AWGN channel, nobody reported this before for bursty impulsive noise channel.

Fig. 5 compares the BER performances of DT with SR scheme assuming $M=3$ relays. For the simulation results the following cases are considered: (1)- the destination has perfect knowledge about $\theta_{m}$ and is utilized in the detection process using (7) and (2)- when $\theta_{m}$ is not utilized by the destination and the LLR values are obtained using the first two terms of (7). From Fig. 5, it is verified that by utilizing $\theta_{m}$ at the destination, our proposed MAP receiver performs significantly better than the case when $\theta_{m}$ is not utilized. This confirms the benefit of exploiting the relay induced BER at the destination for multi-relay scheme. From Fig. 5 it is further verified that similar to SDFR, in case of SR, the optimal MAP receiver performs significantly better than the memoryless receiver when both utilizes $\theta_{m}$ at the destination.

So far it is assumed that the noise samples affecting the relays are spatially independent. On the other hand, we may consider the spatially dependent noise scenario also when all the relays are affected by the same impulsive noise. This is a reasonable assumption [9] to the scenario where all the relays will operate in the same frequency band and hence will be affected by the same interference. In Fig. 6, we demonstrate the effect of spatial dependence on the BER performance. From the figure, it is seen that the performance of the optimal MAP receiver remains unchanged under spatially dependent impulsive noise scenario. 


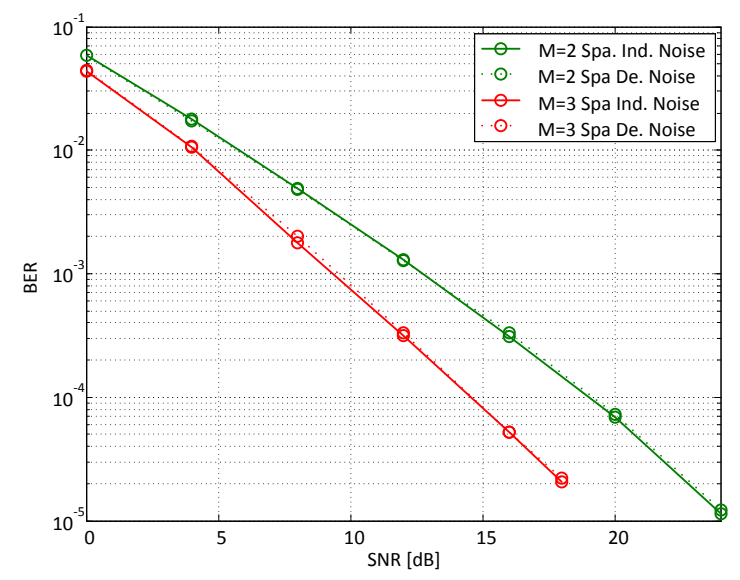

Fig. 6. Effect of spatially dependent impulsive noise on the BER performance of SDFR scheme with $M=2,3$ relays. A BPSK modulation is adopted and each channel is characterized by $p_{B}=0.1, \gamma=100$, and $R=100$ is shown.

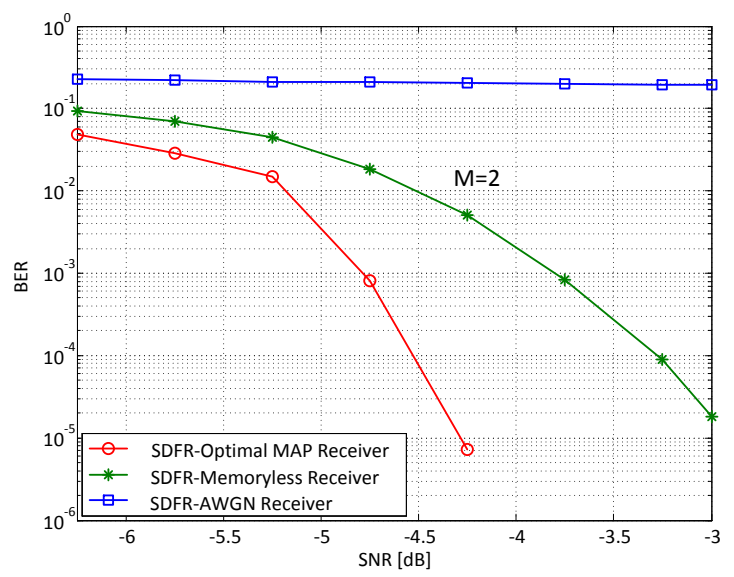

Fig. 7. BER performances of coded SDFR scheme with $M=2$ relays. A BPSK modulation is adopted and each channel is characterized by $p_{B}=0.1$, $\gamma=100, R=100$ is shown.

We also considered systems employing powerful channel codes such as low-density parity check (LDPC) codes. Fig. 7 shows the simulated BER performances of SDFR scheme with $M=2$ relays for LDPC coded transmission assuming three different detectors at the receiver side. It is assumed that a sequence of information bits of length 32,400 is first encoded using LDPC channel coding based on the DVB-S2 standard with the code rate of $1 / 2$. The coded sequence is then interleaved using a random interleaver and mapped onto BPSK modulation sequence. For LDPC decoding, the number of iterations is set to 50. As expected, from Fig. 7, it is observed that similar to uncoded transmission, significant performance gain is achieved when the noise memory is taken into account in the detection process.

\section{CONCLUSION}

In this paper, we have presented the mathematical model to verify the analytical and simulated performances for multirelay DF CR schemes over correlated impulsive noise channel in the presence of Rayleigh fading. From the obtained results, it is observed that the analytical results agree with the simulations and our proposed MAP receiver achieves the LB derived for multi-relay DF CR scheme, and performs significantly better than the conventional schemes that neglect the noise memory. We also have investigated the effect of spatially dependent noise scenario. It is observed that the performance of the optimal MAP receiver remains unchanged under that scenario. Additionally, for SR scheme, by utilizing the relay induced BER at the destination the optimal MAP receiver performs significantly better than the case when the receiver does not have any knowledge about the error at the relay.

\section{ACKNOWLEDGMENT}

This work was supported by Hydro-Quebec, the Natural Sciences and Engineering Research Council of Canada, and McGill University in the framework of the NSERC/HydroQuebec/McGill Industrial Research Chair in Interactive Information Infrastructure for the Power Grid.

\section{REFERENCES}

[1] J. N. Laneman, D. N. Tse, and G. W. Wornell, "Cooperative diversity in wireless networks: Efficient protocols and outage behavior," IEEE Transactions on Infor. Theory, vol. 50, no. 12, pp. 3062-3080, 2004.

[2] D. Middleton, "Statistical-physical models of electromagnetic interference," IEEE Transactions on Electromagnetic Compatibility, vol. EMC19, no. 3, pp. 106-127, 1977.

[3] F. Sacuto, B. L. Agba, F. Gagnon, and F. Labeau, "Evolution of the RF characteristics of the impulsive noise in high voltage environment," in IEEE Third International Conference on Smart Grid Communications (SmartGridComm). IEEE, 2012, pp. 686-691.

[4] S. Al-Dharrab and M. Uysal, "Cooperative diversity in the presence of impulsive noise," IEEE Transactions on Wireless Communications, vol. 8, no. 9, pp. 4730-4739, 2009.

[5] R. Savoia and F. Verde, "Performance analysis of decode-and-forward relaying in impulsive noise environments," in 8th International Symposium on Wireless Communication Systems (ISWCS). IEEE, 2011, pp. 412-416.

[6] A. Nasri and R. Schober, "Performance of cooperative diversity systems in non-gaussian environments," in IEEE International Conference on Communications (ICC). IEEE, 2010, pp. 1-6.

[7] H. Van Khuong and T. Le-Ngoc, "Performance of decode-and-forward cooperative relaying over rayleigh fading channels with impulsive noise," in International Conference on Advanced Technologies for Communications (ATC). IEEE, 2010, pp. 183-188.

[8] M. Ghosh, "Analysis of the effect of impulse noise on multicarrier and single carrier qam systems," IEEE Transactions on Communications, vol. 44, no. 2, pp. 145-147, 1996.

[9] C. Tepedelenlioglu and P. Gao, "On diversity reception over fading channels with impulsive noise," IEEE Transactions on Vehicular Technology, vol. 54, no. 6, pp. 2037-2047, 2005.

[10] M. S. Alam and F. Labeau, "Performance analysis of DF cooperative relaying over bursty impulsive noise channel," in IEEE Sixth International Conference on Smart Grid Communications (SmartGridComm). IEEE, 2015, p. Accepted for publication.

[11] D. Fertonani and G. Colavolpe, "On reliable communications over channels impaired by bursty impulse noise," IEEE Transactions on Communications, vol. 57, no. 7, pp. 2024-2030, 2009.

[12] D. Liang, S. X. Ng, and L. Hanzo, "Relay-induced error propagation reduction for decode-and-forward cooperative communications," in IEEE Global Telecommunications Conference (GLOBECOM 2010). IEEE, 2010, pp. 1-5.

[13] A. K. Sadek, W. Su, and K. R. Liu, "Multinode cooperative communications in wireless networks," IEEE Transactions on Signal Processing, vol. 55, no. 1, pp. 341-355, 2007.

[14] J. G. Proakis, Digital Communications, 4th edition. McGraw-Hill, 2001. 